\title{
Aberrant modulations of static functional connectivity and dynamic functional network connectivity in chronic migraine
}

\author{
Yan Zou ${ }^{1}$, Weijun Tang ${ }^{2}$, Xiangyang Qiao ${ }^{3}, \mathrm{Ji} \mathrm{Li}^{1}$ \\ ${ }^{1}$ Department of Integrated Traditional and Western Medicine, Huashan Hospital, Fudan University, Shanghai, China; ${ }^{2}$ Department of Radiology, \\ Huashan Hospital, Fudan University, Shanghai, China; ${ }^{3}$ Department of Neurology, Huashan Hospital, Fudan University, Shanghai, China \\ Correspondence to: Ji Li. Department of Integrated Traditional and Western Medicine, Huashan Hospital, Fudan University, 12 Middle Urumqi Road, \\ Jing'an District, Shanghai 200040, China. Email: liji_2016@163.com.
}

Background: Chronic migraine (CM) is a common and disabling neurological disorder that affects $1-2 \%$ of the global population. The aim of the present study was to identify the functional characteristics of the CM brain using static functional connectivity (s-FC), static functional network connectivity (s-FNC), and dynamic functional network connectivity (d-FNC) analyses.

Methods: In the present study, 17 CM patients and 20 sex- and age-matched healthy controls (HCs) underwent resting-state functional magnetic resonance imaging. We utilized independent component (IC) analysis to identify 13 ICs. These 13 ICs were then classified into the following 6 resting-state networks (RSNs): the default mode network (DMN), executive control network (ECN), dorsal attention network, auditory network (AN), visual network (VN), and cerebellum network. Subsequently, s-FC, s-FNC, and d-FNC analyses of 13 ICs were employed for between-group comparisons. Three temporal metrics (fraction of time spent, mean dwell time, and number of transitions), which were derived from the state-transition vector, were calculated for group comparisons. In addition, correlation analyses were performed between these dynamic metrics and clinical characteristics [mean visual analog scale (VAS) scores, days with headache per month, days with migraine pain feature per month, and disease duration].

Results: In the comparison of s-FC of 13 ICs within RSNs between the CM and HC groups, increased connectivity was observed in the left angular gyrus (Angular_L) of the ECN (IC 2) and the right superior parietal gyrus (Parietal_Sup_R) of the AN (IC 5), and reduced connectivity was found in the left superior frontal gyrus (Frontal_Sup_2_L) of the AN (IC 5) and DMN (IC 19), the right calcarine sulcus (Calcarine_ R) of the VN (IC 7), and the left precuneus (Precuneus_L) of the DMN (IC 17) in CM patients. In the comparison of the d-FNC of 13 IC pairs within RSNs between the two groups, the CM group exhibited significantly decreased connections between the DMN (IC 11) and AN (IC 5), and increased connections between the ECN (IC 2, IC 4) and DMN (IC 19), ECN (IC 4) and AN (IC 5), and ECN (IC 4) and VN (IC 13) in state 1 . However, no significant differences in s-FNC were observed between the two groups during the s-FNC analysis. Between-group comparisons of three dynamic metrics between the CM and HC groups showed a longer fraction of time spent and mean dwell time in state 2 for CM patients. Furthermore, from the correlation analyses between these metrics and clinical characteristics, we observed a significant positive correlation between the number of transitions and mean VAS scores.

Conclusions: Our findings suggest that functional features of the CM brain may fluctuate over time instead of remaining static, and provide further evidence that migraine chronification may be related to abnormal pattern connectivity between sensory and cognitive brain networks.

Keywords: Chronic migraine; independent component analysis; dynamic functional network connectivity (d-FNC); static functional network connectivity (s-FNC); static functional connectivity (s-FC)

Submitted Apr 21, 2020. Accepted for publication Dec 23, 2020.

doi: 10.21037/qims-20-588

View this article at: http://dx.doi.org/10.21037/qims-20-588 


\section{Introduction}

Chronic migraine $(\mathrm{CM})$, which affects $1-2 \%$ of the global population, is a clearly defined subtype of common chronic daily headache disorder featured by frequent headache attacks occurring on 15 or more days per month (1). It is estimated that approximately $3 \%$ of episodic migraine patients progress into $\mathrm{CM}$ annually, causing substantial social and economic burden $(2,3)$. Aside from the typical migraine profile, CM patients often report other neurological symptoms, such as dizziness, tinnitus, and cognitive impairments of memory and attention $(4,5)$. While the detailed pathophysiology of migraine chronification remains unclear, accumulating evidence suggests that $\mathrm{CM}$ may be associated with functional and structural alterations in brain regions involved in pain-facilitating and paininhibiting processes (6-8).

Resting-state functional magnetic resonance imaging (rs-fMRI) offers a non-invasive method for researchers to probe functional alterations of the human brain (9). Relevant functional neuroimaging studies have shown that cortical and subcortical region alterations are associated with CM, yet most of these studies have focused on static functional connectivity (s-FC) or functional network connectivity. Androulakis et al. observed decreased overall resting-state functional connectivity of the default mode network (DMN), salience network, and executive control network (ECN) in CM patients compared to healthy controls (HCs), indicating that the connectivity of these intrinsic networks may be involved in the development and maintenance of migraine chronification $(10,11)$. In a comparative study, Coppola et al. revealed that functional network connectivity was reduced between the DMN and $\mathrm{ECN}$, and was enhanced between the dorsal attention system and DMN in CM patients (12). Most of previous rsfMRI studies assume that functional connections between brain areas remain relatively stable over time (13). However, emerging evidence suggests that the brain is a complex system with time-dependent and dynamic characteristics, where connections between brain areas may constantly vary over time $(14,15)$. A recent technique, called "dynamic connectivity analysis", reflects the time-varying properties of connectivity by dividing resting-state time series into multiple overlapping "sliding windows" (16). Analyses of these sliding windows can identify functional connectivity features on the order of seconds rather than minutes, and can further help to identify significant networks that are not detected by static analysis (17).
Inspired by previous work, we combined conventional s-FC, static functional network connectivity (s-FNC), and novel dynamic functional network connectivity (d-FNC) analyses to investigate the functional connectivity features in CM. The aim of the present study was primarily two-fold: we sought to explore whether dysfunctional brain regions or brain networks in CM patients were similar to those previously reported in the literature, and whether dynamic properties of altered brain networks were associated with clinical variables.

\section{Methods}

\section{Participants}

Seventeen CM patients (10 females and 7 males, aged $45.41 \pm 14.87$ years) were recruited and met the following criterion outlined by the International Classification of Headache Disorders III: headaches occurring 15 or more days per month for 3 months or longer with 8 or more days having the features of migraine pain. Before resting-state scanning, each CM patient was required to keep a 4-week headache diary, including headache days, migraine days, and headache severity. CM patients were excluded if they exhibited the following characteristics: (I) clinical headache severity score $<4$ [visual analog scale (VAS) score range $0-10]$; (II) lack of compliance in completing the headache diary; (III) other neurological or psychiatric disorders; (IV) history of head trauma; (V) contraindication for MRI scanning; (VI) simultaneous participation in other clinical trials; or (VII) overuse of pain medication. All CM patients were scanned during baseline pain or at least 24 hours outside of their headache attack phases.

In addition, 20 age-, sex-, and handedness-matched HCs (11 females and 9 males, aged $43.56 \pm 8.77$ years) were recruited. None of the HCs had a history of chronic illness, such as hypertension or diabetes, or a family history of migraine. The present study was approved by the Ethics Committee of Huashan Hospital of Fudan University (no. 2019-M012), and written informed consent was obtained from all participants.

\section{Assessment of clinical characteristics}

Mean headache severity per month (mean VAS scores 0-10), days with headache per month, days with migraine pain feature per month, and disease duration (months) were calculated for each patient according to the 4-week 
headache diary.

\section{Data acquisition and preprocessing}

The fMRI data were acquired using a GE Signa VH/i 3.0T scanner (General Electric, Milwaukee, Michigan, USA) with an 8-channel phased-array head coil. Participants were instructed to relax while awake, with their eyes closed. The imaging parameters have been previously described (7). Briefly, the $\mathrm{T} 2{ }^{*}$-weighted echo-planar image sequence included the following: repetition time $(\mathrm{TR})=2,000 \mathrm{~ms}$, time to echo $(\mathrm{TE})=35 \mathrm{~ms}$, flip angle $=90^{\circ}$, matrix $=64 \times 64$, field of view $=256$, slice thickness $=4 \mathrm{~mm}$, gap $=0 \mathrm{~mm}$, and 200 time points.

Preprocessing was performed with the Data Processing \& Analysis for Brain Imaging toolbox (http://rfmri.org/ dpabi) as follows: (I) removal of the first 10 volumes; (II) slice-timing correction; (III) head motion correction; (IV) co-registration to the respective T1-anatomical image for each participant; (V) spatial normalization to the Montreal Neurological Institute (MNI) space; (VI) resampling of 3-mm cubic voxels; and (VII) spatial smoothing of 6-mm full-width, half-maximum isotropic Gaussian kernel.

\section{Identification of intrinsic connectivity networks}

Independent component analysis (ICA), which was implemented using the Group ICA Of fMRI Toolbox (GIFT) software (http://mialab.mrn.org/software/gift), was used to identify intrinsic connectivity networks. The procedure consisted of the following steps: (I) independent component (IC) estimation with minimum description length (MDL) criteria (18); (II) IC separation using the infomax algorithm; (III) back reconstruction of each IC; (IV) specific resting-state network (RSN) map selection from all ICs; and (V) z-score transformation of each IC spatial map. Finally, 20 ICs were estimated based on the MDL criteria, and 13 ICs were identified as meaningful via visual inspection. We sorted these 13 ICs into the following 6 RSNs for subsequent analyses: the DMN, ECN, dorsal attention network (DAN), auditory network (AN), visual network $(\mathrm{VN})$, and cerebellum network $(\mathrm{CN})$.

\section{Computation for s-FNC}

The s-FNC analysis was conducted using the MANCOVAN toolbox in GIFT software to explore changes in functional connectivity within the predefined 13 spatial IC pairs. First, the time courses of selected ICs were detrended, despiked, and low-pass filtered at $0.01-0.15 \mathrm{~Hz}$. Subsequently, pairwise correlations of these ICs were computed and then transformed using the Fisher's Z-transformation.

\section{Computation for d-FNC}

The d-FNC analysis was conducted using the sliding window approach with the temporal d-FNC toolbox in GIFT software $(16,19)$. The sliding-window approach exposes time-varying patterns of functional connectivity within the 13 IC pairs over the scan duration. First, the time courses of selected IC pairs were detrended, despiked, and filtered at $0.15 \mathrm{~Hz}$. Next, a window size of 30 TRs sliding in steps of 1 TR convolution and a Gaussian distribution ( $\sigma=3$ TRs) was applied to divide the time courses of all IC pairs. Therefore, 198 windows in total were obtained for each participant to capture the time variability in connectivity between all pairwise ICs. Subsequently, the optimal number of clusters was estimated to be 6 by conducting k-means analyses using an elbow criterion. Therefore, k-means clustering $(\mathrm{k}=6)$ with city distance was performed to separate all obtained windows (37 participants $\times 198$ windows $=7,326$ matrices) into six clusters, representing six dynamic states across the resting-state scan period. In addition, three temporal metrics (the fraction of time spent in each state, mean dwell time in each state, and the number of transitions changes among different states) were calculated for each participant.

\section{Statistical analysis}

\section{Analysis of imaging data}

For the s-FC analysis, one-sample $t$-tests were performed to determine each IC map of the CM and HC groups. Two-sample $t$-tests were performed to compare intergroup differences within each IC map between the CM and HC groups. The threshold for statistical significance was set at a $P$ value $<0.05$ (AlphaSim correction).

For the s-FNC analysis, two-sample $t$-tests were performed to detect pairwise differences in functional network connectivity of 13 IC pairs between the HC and $\mathrm{CM}$ groups. The threshold for statistical significance was set at a $\mathrm{P}$ value $<0.05$ [false discovery rate (FDR) correction].

For the d-FNC analysis, two-sample $t$-tests were performed to detect differences in functional network connectivity of 13 IC pairs between two groups in each state. The threshold for statistical significance was set at a $\mathrm{P}$ 
Table 1 Clinical details of the CM and HC groups

\begin{tabular}{lcc}
\hline Variables & CM group & HC group \\
\hline No. of cases & 17 & 20 \\
Males, $\mathrm{n}$ & 7 & 11 \\
Females, $\mathrm{n}$ & $45.41 \pm 14.87$ & $4.92 \pm 1.21$ \\
Age (years), mean \pm SD & $56 \pm 8.77$ \\
Mean headache severity per month (VAS score 0-10), mean \pm SD & $17.76 \pm 2.19$ & - \\
Days with headache per month, mean \pm SD & $10.65 \pm 2.83$ & - \\
Days with migraine pain feature per month, mean \pm SD & $10.47 \pm 3.99$ & - \\
Disease duration (months), mean \pm SD &
\end{tabular}

Results represent mean \pm standard deviation (SD). CM, chronic migraine; HC, healthy control; VAS, visual analog scale.

value $<0.05$ (FDR correction).

\section{Analysis of clinical data}

Non-parametric tests were performed to compare changes in three temporal metrics (fraction of time spent, mean dwell time, and number of transitions) between the CM and HC groups.

Mean VAS scores, days with headache per month, days with migraine pain feature per month, and disease duration (months) were used to perform correlation analyses with the three temporal metrics in CM patients.

\section{Results}

\section{Clinical characteristics for all participants}

The clinical characteristics of all participants are shown in Table 1. No apparent differences were found in age or sex between the $\mathrm{CM}$ and $\mathrm{HC}$ groups $(\mathrm{P}>0.05)$.

\section{RSNS}

A total of 20 ICs were obtained by ICA IC analysis, and 13 ICs were identified as meaningful. These 13 ICs were then grouped into the following six RSNs: DMN (IC 1/11/17/19), ECN (IC 2/4/9), DAN (IC 3), AN (IC 5), VN (IC 7/13), and CN (IC 16/18) (Figure 1A).

Group mean s-FNC between IC pairs within RSNs computed over the entire scan is shown in Figure $1 B$. Each of the 13 ICs was rearranged according to the 6 functional network groups (DMN, ECN, DAN, AN, VN, or CN). Patterns of functional network connectivity resembled findings from prior literature $(19,20)$, showing that the network components were highly correlated within the RSN groups, especially within the DMN, ECN, and VN. Furthermore, the ECN was correlated with DMN, and both correlations and anticorrelations were observed between $\mathrm{VN}$ and $\mathrm{CN}$.

\section{s-FC of IC maps within RSNs}

Comparison of intrinsic connectivity of ICs within RSNs revealed that the $\mathrm{CM}$ group showed significantly increased functional connectivity in the ECN [IC 2: left angular gyrus (Angular_L) MNI coordinates x, y, z: $-54,-57,24[$ and AN [IC 5: right superior parietal gyrus (Parietal_Sup_R), MNI coordinates x, y, z: 24, -54, 60]. In addition, decreased functional connectivity in the AN [IC 5: left superior frontal gyrus (Frontal_Sup_2_L), MNI coordinates x, y, z: $-24,30,36$ ]; VN [IC 7: right calcarine sulcus (Calcarine_R), MNI coordinates $\mathrm{x}, \mathrm{y}, \mathrm{z}: 6,-72,6]$, and DMN [IC 17: left precuneus (Precuneus_L), MNI coordinates x, y, z: 0, -57 , 15; IC 19: left superior frontal gyrus (Frontal_Sup_2_L), MNI coordinates $\mathrm{x}, \mathrm{y}, \mathrm{z}:-21,54,15]$ were also observed in the CM group (Figures 2,3; Table 2).

\section{s-FNC strength}

There was no significant difference in functional network connectivity of IC pairs within RSNs between the CM and HC groups.

\section{d-FNC states and connectivity strength}

The time-varying functional network connectivity between 13 IC pairs within RSNs during the whole scan duration can 

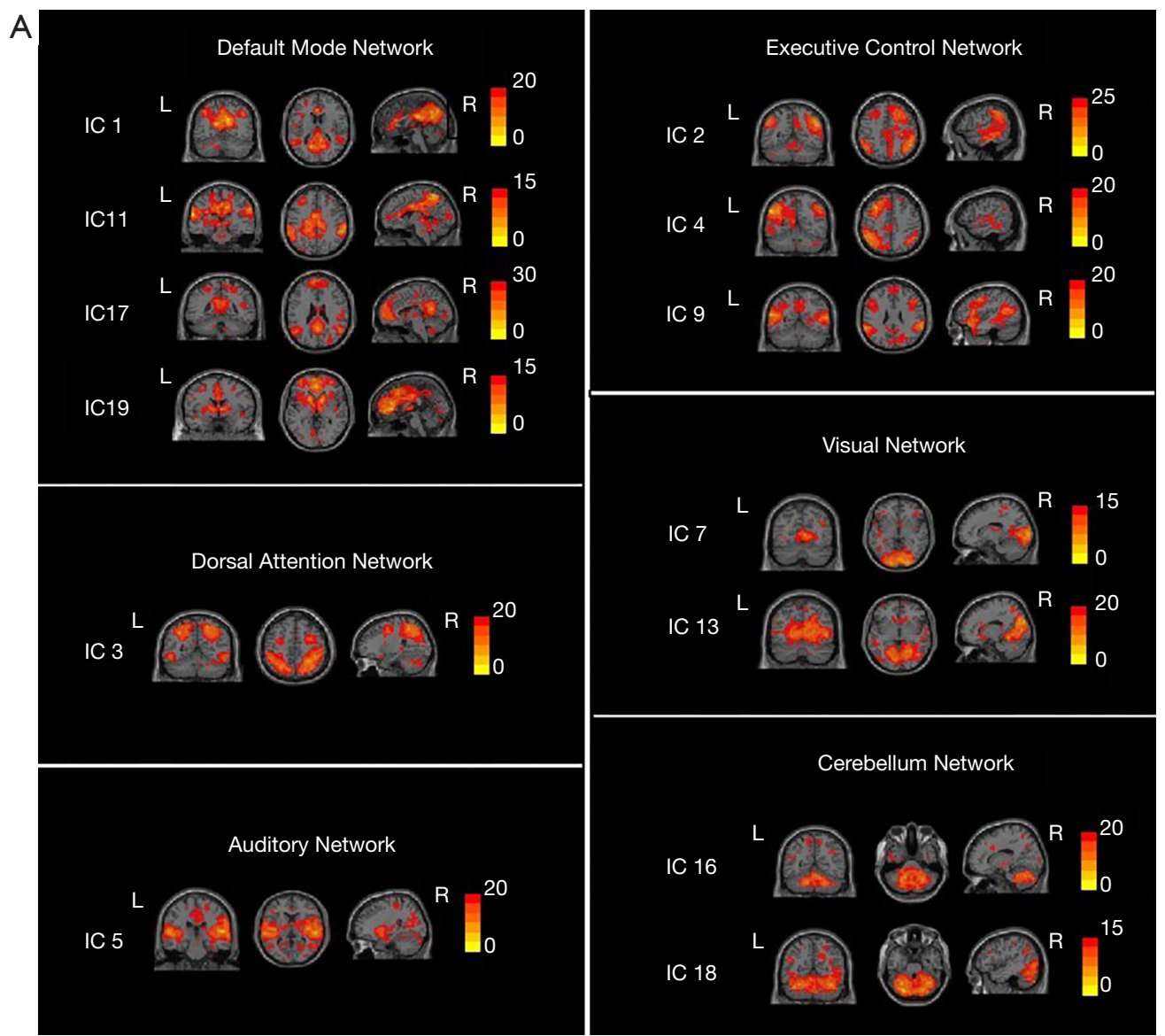

B

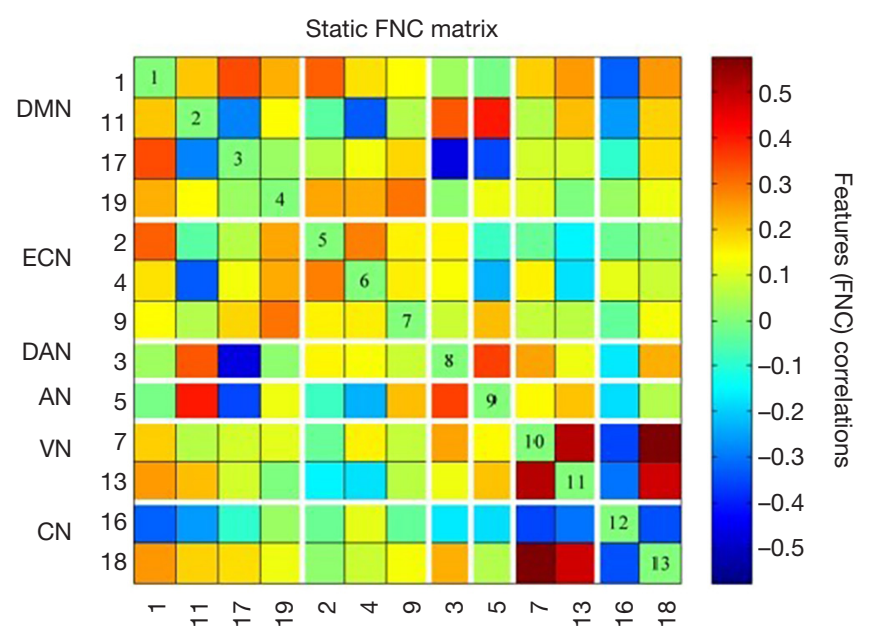

Figure 1 Functional connectivity within and between the independent components (ICs) of resting-state networks (RSNs). (A) Spatial mapping of the 13 ICs identified six RSNs: the default mode network (DMN; IC 1/11/17/19), executive control network (ECN; IC 2/4/9), dorsal attention network (DAN; IC 3), auditory network (AN; IC 5), visual network (VN; IC 7/13), and cerebellum network (CN; IC 16/18). (B) Group mean static functional network connectivity (FNC) between the ICs within RSNs. 


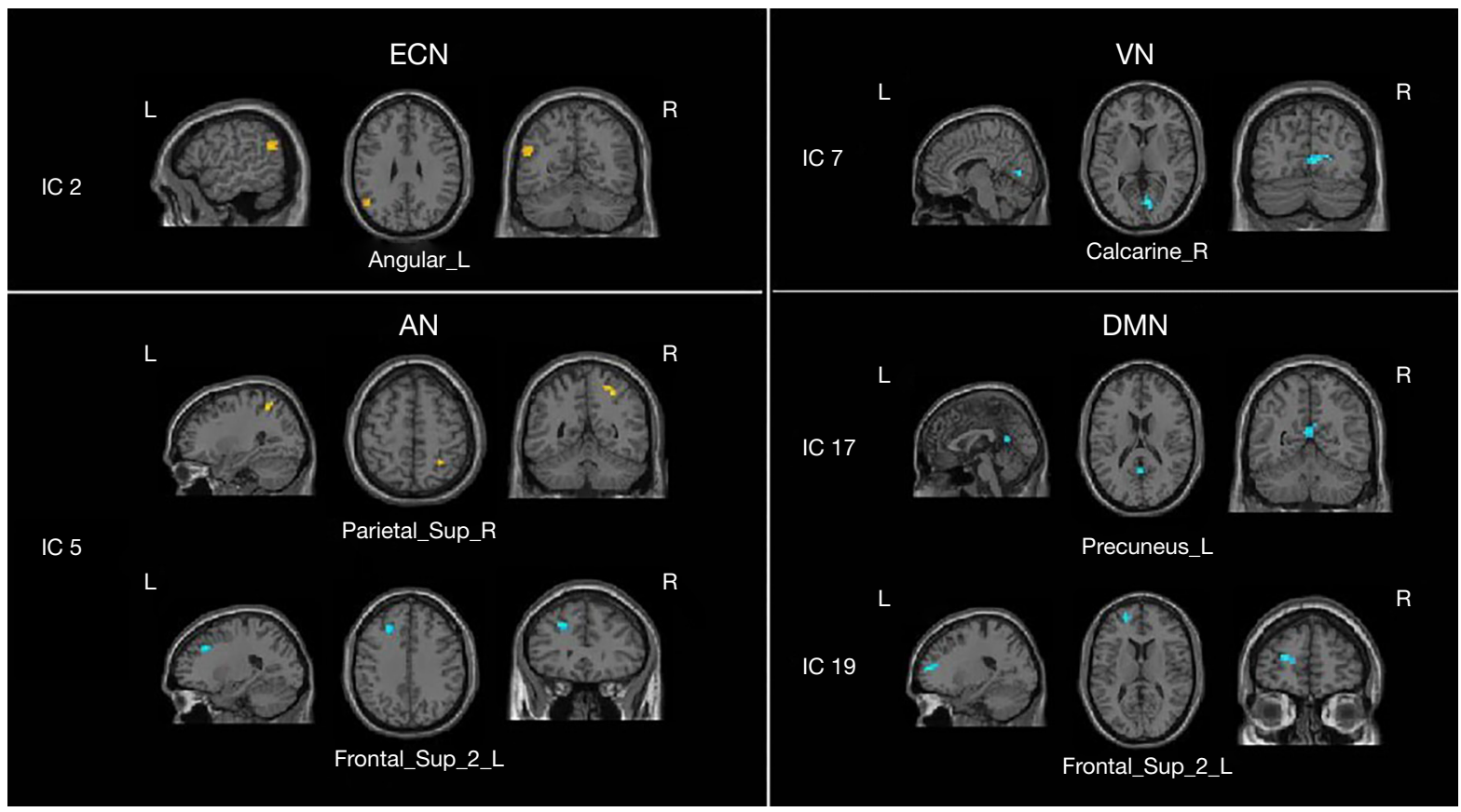

Figure 2 Spatial maps of differences of independent components (ICs) within resting-state networks (RSNs) between the chronic migraine $(\mathrm{CM})$ and healthy control $(\mathrm{HC})$ groups $(\mathrm{P}<0.05$, AlphaSim corrected). Compared with the HC group, the CM group demonstrated increased connectivity in the executive control network (ECN; IC 2: Angular_L) and auditory network (AN; IC 5: Parietal_Sup_R), and decreased connectivity in the AN (IC 5: Frontal_Sup_2_L), visual network (VN; IC 7: Calcarine_R), and the default mode network (DMN; IC 17: Precuneus_L, IC 19: Frontal_Sup_2_L) $(\mathrm{P}<0.05$, AlphaSim corrected). Angular_L, the left angular gyrus; Parietal_Sup_R, right superior parietal gyrus; Frontal_Sup_2_L, the left superior frontal gyrus; Calcarine_R, the right calcarine sulcus; Precuneus_L, the left precuneus.

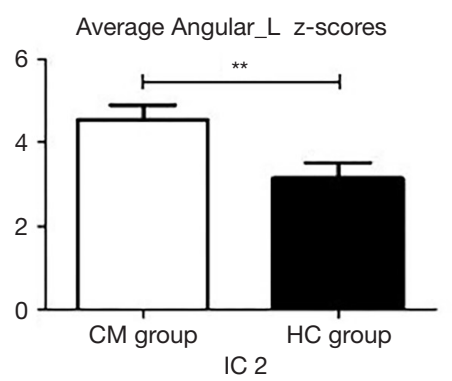

Average Calcarine_R z-scores

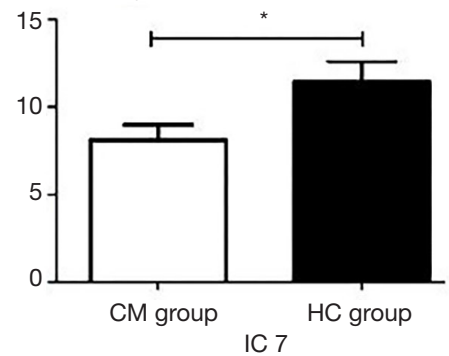

Average Parietal_Sup_R z-scores

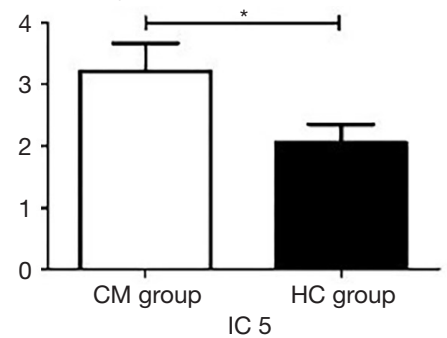

Average Precuneus_L z-scores

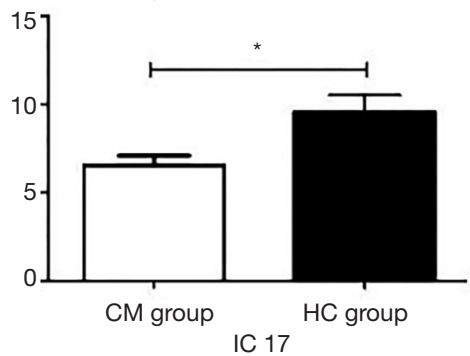

Average Frontal_Sup_2_L z-scores

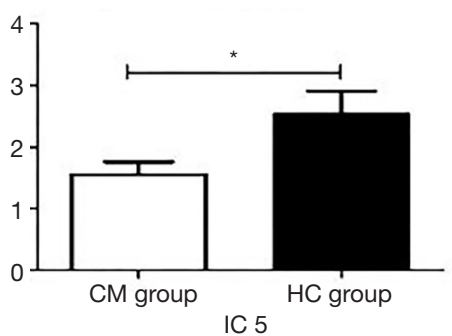

Average Frontal_Sup_2_L z-scores

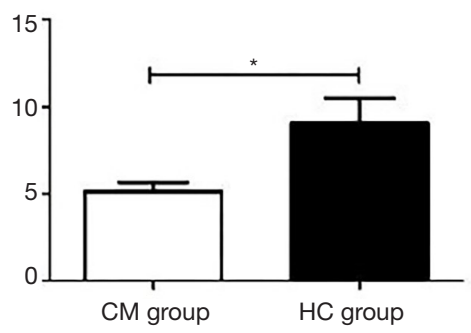

IC 19

Figure 3 Bar graphs of the mean z-scores of altered regions of independent components (ICs) within resting-state networks (RSNs) between the chronic migraine (CM) and healthy control (HC) groups. Asterisks denote statistically significant differences between the two groups. Error bars represent standard error. *, $\mathrm{P}<0.05 ;{ }^{* *}, \mathrm{P}<0.01$. 
Table 2 Significantly altered IC maps in the CM group compared with the HC group

\begin{tabular}{|c|c|c|c|c|c|c|c|}
\hline IC & Sign & Region label & Extent & t-value & \multicolumn{3}{|c|}{ MNI coordinates } \\
\hline 2 & Positive & Angular_L & 42 & 6.26 & -54 & -57 & 24 \\
\hline \multirow{2}{*}{5} & Positive & Parietal_Sup_R & 23 & 4.97 & 24 & -54 & 60 \\
\hline & Negative & Frontal_Sup_2_L & 23 & -5.16 & -24 & 30 & 36 \\
\hline 17 & Negative & Precuneus_L & 30 & -5.09 & 0 & -57 & 15 \\
\hline 19 & Negative & Frontal_Sup_2_L & 27 & -4.45 & -21 & 54 & 15 \\
\hline
\end{tabular}

$\mathrm{CM}$, chronic migraine; HC, healthy control; IC, independent component; MNI, Montreal Neurological Institute.

be represented by six states (Figure 4). The cluster number and percentage of scan time spent by all participants in each state are shown in Figure 4A. Group-specific centroids for each state of the CM and $\mathrm{HC}$ groups are shown in Figure $4 B, C$. Matrix plots of significant functional network connectivity differences between the $\mathrm{CM}$ and $\mathrm{HC}$ groups were observed in state $1(\mathrm{P}<0.05$, FDR corrected), as shown in Figure 5. Compared to the HC group, the CM group exhibited significantly decreased connections between the DMN (IC 11) and AN (IC 5), and increased connections between the ECN (IC 2, IC 4) and DMN (IC 19), the $\mathrm{ECN}$ (IC 4) and AN (IC 5), and the ECN (IC 4) and VN (IC 13) in state 1. There were no significant differences in functional network connectivity in the other states.

\section{Correlation between clinical characteristics and temporal metrics}

A significantly longer fraction of time spent and mean dwell time were found in the $\mathrm{CM}$ group versus the $\mathrm{HC}$ group in state 2. However, there was no significant difference in the number of transitions between two groups (Figure 6A,B,C). In further analyses of correlations between these three temporal properties and clinical characteristics [mean VAS scores, days with headache per month, days with migraine pain feature per month, and disease duration (months)] in CM patients, the number of transitions was positively correlated with mean VAS scores. However, these temporal properties and other clinical characteristics were not statistically significant (Figure 6D).

\section{Discussion}

To the best of our knowledge, the present study is the first to combine s-FC, s-FNC, and d-FNC analyses to investigate the whole-brain features of CM. Our work revealed the following findings: (I) dysfunctional intrinsic connectivity of brain regions involved in the ECN (Angular_L), DMN (Precuneus_L, Frontal_Sup_2_L), AN (Parietal_Sup_R, Frontal_Sup_2_L), and VN (Calcarine_ $\mathrm{R})$ were observed in $\mathrm{CM}$ patients, and these pronounced abnormalities were also found in previous studies (21); (II) according to s-FNC analysis, no obvious s-FNC differences were found between the CM and HC groups; (III) statespecific dysfunctional functional network connectivity patterns were observed between the DMN and $\mathrm{AN}$, the $\mathrm{ECN}$ and DMN, the ECN and AN, and the ECN and VN in state 1 in the CM group during d-FNC analysis; (IV) altered temporal metrics, including the fraction of time spent and mean dwell time, were found in state 2 in the $\mathrm{CM}$ group; and (V) a significant positive correlation between the number of transitions and mean VAS scores was observed in CM patients.

In the comparison of s-FC within the six separated RSNs between the CM and $\mathrm{HC}$ groups, our findings were in line with those previous studies which report dysfunctional brain regions in CM patients to be significantly involved in pain-related functions (e.g., precuneus and prefrontal cortex) (22-24). Chronic pain is considered a dysfunctional process defined by longstanding pain sensations $>3$ months $(25,26)$. Pain experience is a complex process that consists of sensory discrimination, and affective emotional and cognitive evaluation aspects (27-29). Evidence suggests that persistent pain could cause reorganization of intrinsic brain networks, altering the perceptual process of pain $(8,11)$. In our study, the corresponding dysfunctional brain networks in CM patients were primarily involved in sensory networks ( $\mathrm{AN}$ and $\mathrm{VN}$ ), and the cognitive and emotional 


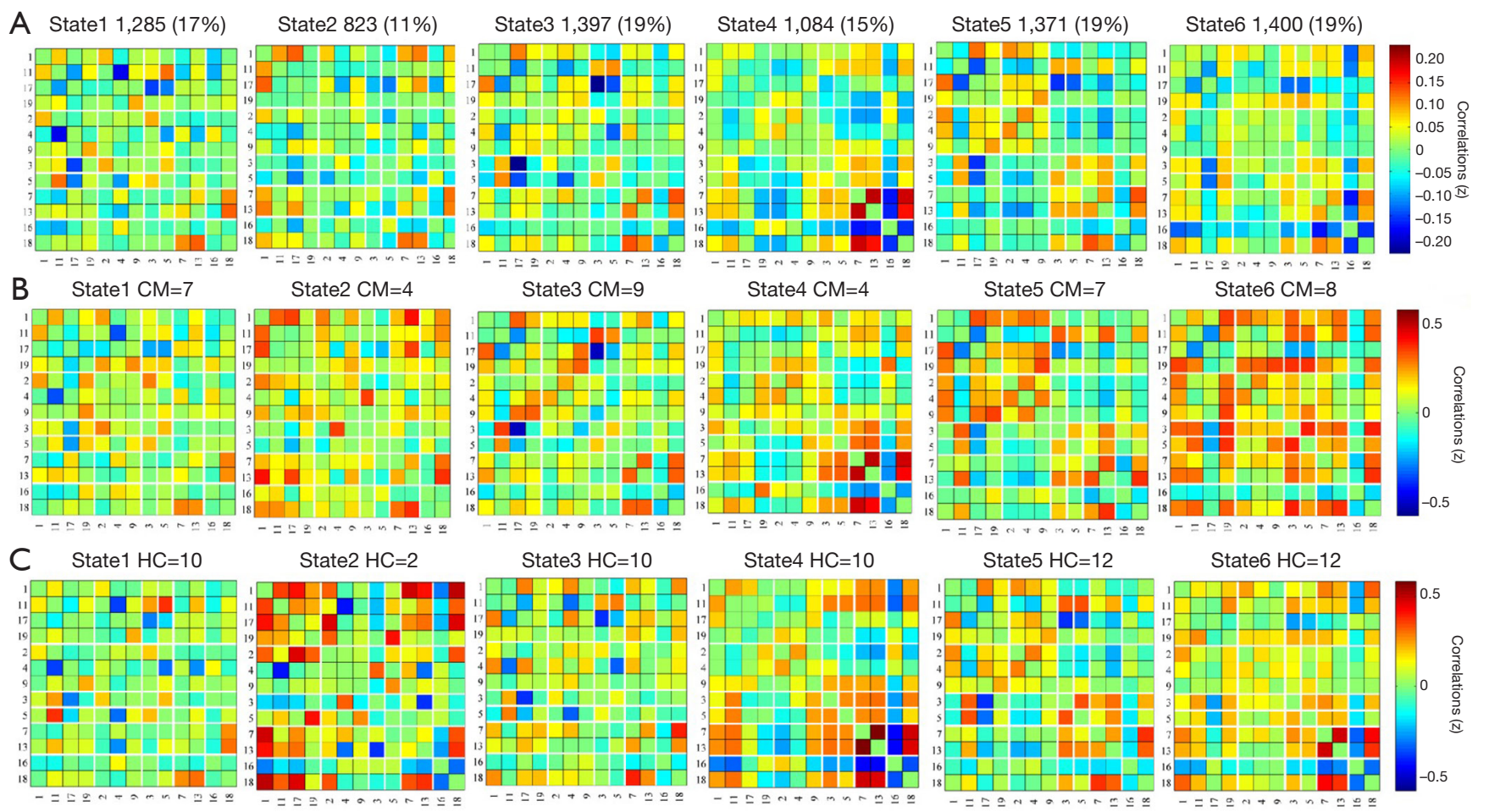

Figure 4 Dynamic functional network connectivity (d-FNC) analysis of independent component (IC) pairs within resting-state networks (RSNs) revealed six brain states. (A) Cluster medians of the six states of the participants are shown, along with the total number and proportion of scan time. (B) Group-specific centroids of the states for the chronic migraine (CM) group. (C) Group-specific centroids of the states for the healthy control (HC) group. The color bar represents the z-values of the functional network connectivity (FNC).

implementation-related networks (DMN and ECN) (30,31). The $\mathrm{VN}$ and $\mathrm{AN}$, as parts of the sensory system, are responsible for processing external stimulus information. Pain experience is normally accompanied by several sensory inputs, such as vision, audition, and olfaction, which may interfere with one another (32-34). Our findings revealed increased connectivity within the $\mathrm{VN}$ and $\mathrm{AN}$, indicating that visual or auditory stimuli might enhance the saliency of painful input, leading to increased pain sensation. Interestingly, decreased connectivity within the AN was also observed in CM patients. It is possible that central sensitization caused by constant noxious input can attenuate the perception of sensory inputs, leading to the neurological symptoms commonly experienced by CM patients, such as phonophobia $(35,36)$.

Although the DMN and ECN both regulate higherorder cognitive functions, the DMN is a "task-negative" network that supports internal mental exploration, while the $\mathrm{ECN}$ is a "task-positive" network that engages in external stimuli and tasks $(12,37)$. Internally self-focused attention is characterized by increased intrinsic DMN connectivity and decreased intrinsic ECN connectivity, whereas externally goal-oriented attention is characterized by decreased intrinsic DMN connectivity and increased intrinsic ECN connectivity $(38,39)$. We posit that the anticorrelation between these two networks reflects a switching balance between internally and externally directed cognition $(40,41)$. Decreased functional connectivity within the DMN and increased functional connectivity within the ECN of CM patients were also observed in our study, verifying the reciprocal inhibitory relationship between the DMN and ECN. We speculate that constant pain acts as an additional cognitive load, further enhancing ECN connectivity. For the DMN, however, painful stimuli attenuate its connectivity.

In comparing static and d-FNC differences within the six RSNs between the CM and HC groups, we found significant between-group functional network connectivity differences among several RSNs, including the DMN, $\mathrm{ECN}, \mathrm{VN}$, and $\mathrm{AN}$, in state 1 of $\mathrm{CM}$ patients using d-FNC 


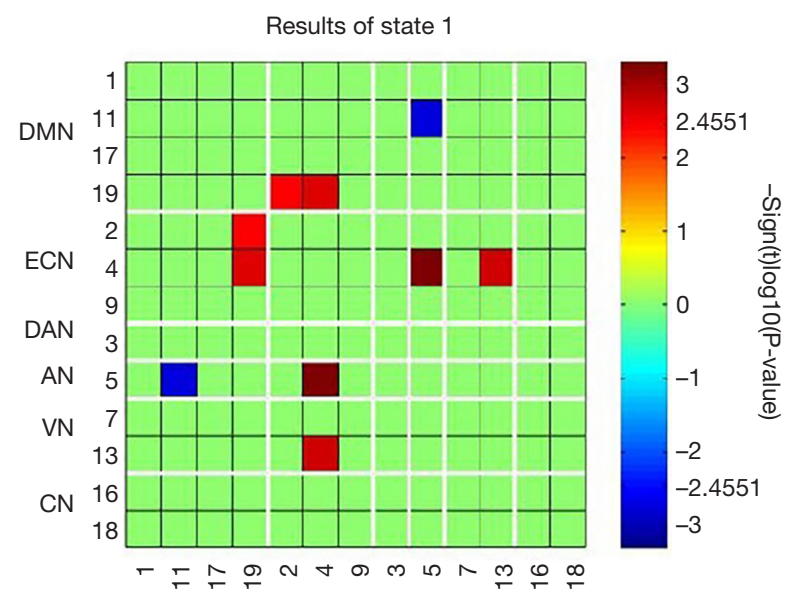

Figure 5 Matrix plot of significant dynamic functional network connectivity (FNC) differences between the chronic migraine (CM) and healthy control $(\mathrm{HC})$ groups $(\mathrm{P}<0.05$, false discovery rate corrected). Warm colors represent significantly increased connections, and the blue colors denote significantly decreased connections in the CM group compared with the HC group. The color bar represents the z-values of the FNC. Based on the anatomical and functional properties of independent components (ICs). ICs 1/11/17/19, ICs 2/4/9, IC 3, IC 5, IC 7/13, and IC 16/18 were categorized into the default mode network (DMN), executive control network (ECN), dorsal attention network (DAN), auditory network (AN), visual network (VN), and cerebellum network $(\mathrm{CN})$, respectively.

A

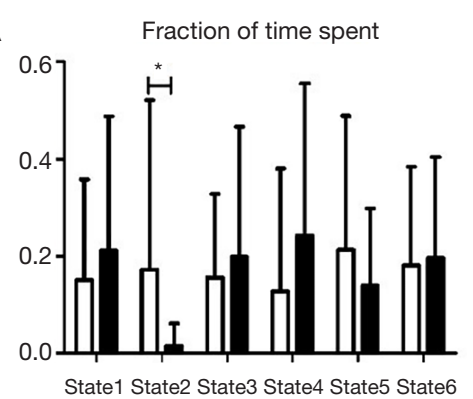

C

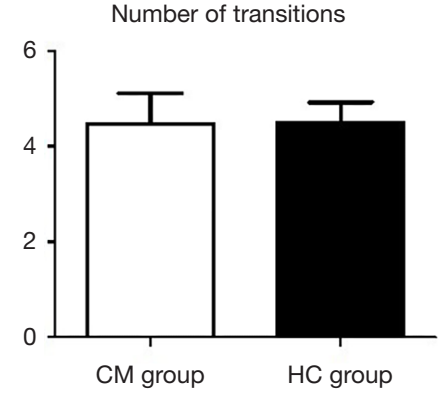

B
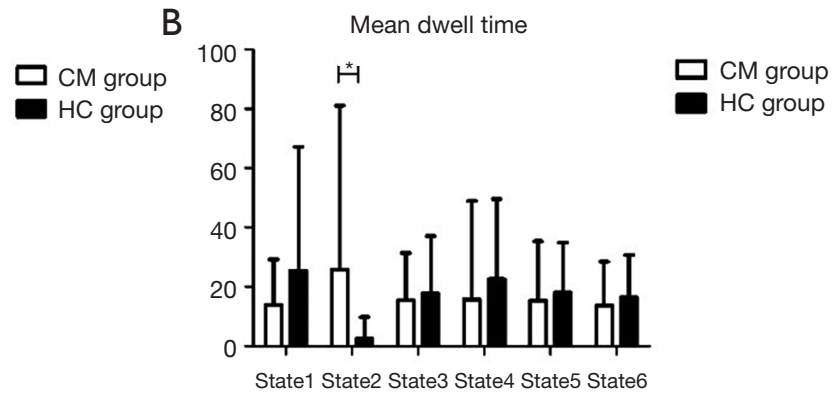

D

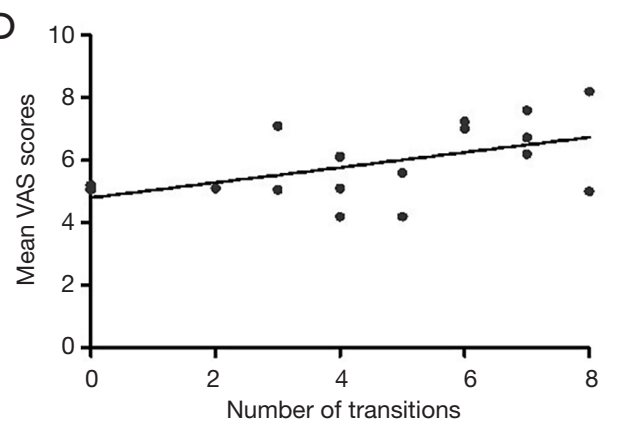

Figure 6 Temporal properties of functional connectivity state analysis. (A,B,C) Bar graphs of the mean dwell time and fraction of time spent, along with the number of transitions between the chronic migraine (CM) and healthy control (HC) groups. (D) Scatter graphs of the number of transitions and mean visual analog scale (VAS) scores. The number of transitions and mean VAS scores of the two patients were similar. Asterisks denote statistically significant differences between the two groups. Error bars represent standard error. *, $\mathrm{P}<0.05$. 
analysis. However, s-FNC analysis revealed no significant difference in functional network connectivity between the two groups. In our study, 6 dynamic states were ultimately defined, and between-group differences were compared within each state by strict correction. Dynamic FNC analysis utilized the temporal information within rs-fMRI data, and between-group comparison was conducted in functional network connectivity matrices of each state, whereas s-FNC analysis only compared the average spatial functional network connectivity matrices of two groups. This might have been the reason why significant group differences were found in the d-FNC analysis, but not in the s-FNC analysis. Of note, the disrupted functional network connectivity in state 1 was primarily related to the ECN, which has been demonstrated to be active during cognitive tasks, such as in goal-directed behavior, decision-making, and working memory $(42,43)$. Several studies focusing on chronic pain have revealed abnormal cortical activity, both increased and decreased, of brain regions within the $\mathrm{ECN}$, and dysregulation of this network is believed to influence perception and corresponding sensory cortical activity $(23,44)$. In the current study, stronger functional connectivity between the ECN and sensory networks (AN and $\mathrm{VN}$ ) may indicate that sustained attention from chronic pain evokes ECN activity, enhances its information communication with sensory systems, disrupts the top-down (sensory behavior) control processes for pain, and leads to cognitive impairments, such as depression. Furthermore, the weaker connectivity observed between the DMN and $\mathrm{VN}$ in our study may also reflect deficits in cognitive and sensory processes in $\mathrm{CM}$ patients.

We also compared dynamic temporal metrics between the $\mathrm{CM}$ and $\mathrm{HC}$ groups. Although the total number of state transitions between the two groups were nearly the same, CM patients spent more time in state 2. Furthermore, exploratory correlation analyses between these dynamic metrics and clinical characteristics were performed, and we observed a positive correlation between the number of transitions and mean VAS scores, suggesting a positive relationship between changes in the number of transitions among different states and CM headache severity. These results suggest that these dynamic measures could be alternative indicators for investigating subtle changes that occur in CM.

\section{Limitations}

The present study has several limitations. First, all CM patients in the study were approved to take painkillers when they experienced unendurable pain. Therefore, our findings might have been affected by the use of these drugs. To minimize this interference, participants who overused pain medication were excluded before the study commenced, and all CM patients underwent MRI scanning at their basal level of pain or at least 24 hours beyond the onset of their acute headache. Second, our study adopted a crosssectional design, and the inference of causal associations from the observed results is limited. Future studies with a longitudinal design to assess the sensitivity of d-FNC analysis during the evolution of $\mathrm{CM}$ are warranted. Third, the sample size of both the CM and HC groups was relatively small. More participants should be recruited to verify the findings of this study.

\section{Conclusions}

The present study represents the first attempt to adopt s-FC, s-FNC, and d-FNC analyses to map functional connections among whole-brain networks in $\mathrm{CM}$ patients. Our results replicated previous work, which demonstrated that dysfunctional s-FC of brain regions for CM patients were mainly involved in sensory and cognitive systems. Furthermore, between-group differences in functional network connectivity across time, as captured by the d-FNC analysis, provide additional valuable information that cannot be found using s-FNC analysis. We found that the abnormal functional network connectivity patterns were state dependent, and altered connectivity was mostly concentrated in the ECN of CM patients using the d-FNC analysis. These findings support the use of novel perspective which may be helpful for further elucidating the functional characteristics of CM brains.

\section{Acknowledgments}

Funding: The present study was sponsored by the Health and Family Planning Commission of Shanghai Municipality (no. 2018LZ001) and the Science and Technology Commission of Shanghai Municipality (no. 18401901900).

\section{Footnote}

Conflicts of Interest: All authors have completed the ICMJE uniform disclosure form (available at http://dx.doi. org/10.21037/qims-20-588). The authors have no conflicts of interest to declare. 
Ethical Statement: The present study was approved by the Ethics Committee of Huashan Hospital of Fudan University (no. 2019-M012), and informed consent was obtained from all the patients.

Open Access Statement: This is an Open Access article distributed in accordance with the Creative Commons Attribution-NonCommercial-NoDerivs 4.0 International License (CC BY-NC-ND 4.0), which permits the noncommercial replication and distribution of the article with the strict proviso that no changes or edits are made and the original work is properly cited (including links to both the formal publication through the relevant DOI and the license). See: https://creativecommons.org/licenses/by-nc-nd/4.0/.

\section{References}

1. Headache Classification Committee of the International Headache Society (IHS). The International Classification of Headache Disorders, 3rd edition (beta version). Cephalalgia 2013;33:629-808.

2. Natoli JL, Manack A, Dean B, Butler Q, Turkel CC, Stovner L, and Lipton RB. Global prevalence of chronic migraine: a systematic review. Cephalalgia 2010;30:599-609.

3. Dodick DW. Migraine. Lancet 2018;391:1315-30.

4. May A, Schulte LH. Chronic migraine: risk factors, mechanisms and treatment. Nat Rev Neurol 2016;12:455-64.

5. Su M, Yu SY. Chronic migraine: A process of dysmodulation and sensitization. Mol Pain 2018;14:1744806918767697.

6. Ho S, Darby D, Bear N. Cognitive effects of onabotulinumtoxinA in chronic migraine. BMJ Neurology Open 2020;2:e000014.

7. Zou Y, Tang WJ, Li X, Xu MW, Li J. Acupuncture Reversible Effects on Altered Default Mode Network of Chronic Migraine Accompanied with Clinical Symptom Relief. Neural Plast 2019;2019:5047463.

8. Kuner R, Flor H. Structural plasticity and reorganisation in chronic pain. Nat Rev Neurosci 2017;18:113.

9. Lu W, Dong KJ, Cui D, Jiao Q, Qiu JF. Quality assurance of human functional magnetic resonance imaging: a literature review. Quant Imaging Med Surg 2019;9:1147-62.

10. Androulakis XM, Krebs KA, Jenkins C, Maleki N, Finkel AG, Rorden C, Newman R. Central Executive and Default Mode Network Intranet work Functional Connectivity Patterns in Chronic Migraine. J Neurol Disord 2018;6:393.

11. Androulakis XM, Krebs K, Peterlin BL, Zhang TM,
Maleki N, Sen S, Rorden C, Herath P. Modulation of intrinsic resting-state fMRI networks in women with chronic migraine. Neurology 2017;89:163-9.

12. Coppola G, Renzo AD, Petolicchio B, Tinelli E, Lorenzo CD, Parisi V, Serrao M, Calistri V, Tardioli S, Cartocci G, Schoenen J, Caramia F, Piero VD, Pierelli F. Aberrant interactions of cortical networks in chronic migraine: A resting-state fMRI study. Neurology 2019;92:e2550-8.

13. Necka EA, Lee IS, Kucyi A, Cheng JC, Yu QB, Atlas LY. Applications of dynamic functional connectivity to pain and its modulation. Pain Rep 2019;4:e752.

14. Calhoun VD, Adali T. Time-Varying Brain Connectivity in fMRI Data: Whole-brain data-driven approaches for capturing and characterizing dynamic states. IEEE Signal Process Mag 2016;33:52-66.

15. Rashid B, Damaraju E, Pearlson GD, Calhoun VD. Dynamic connectivity states estimated from resting fMRI Identify differences among Schizophrenia, bipolar disorder, and healthy control subjects. Front Hum Neurosci 2014;8:897.

16. Allen EA, Damaraju E, Plis SM, Erhardt EB, Eichele T, Calhoun VD. Tracking whole-brain connectivity dynamics in the resting state. Cereb Cortex 2014;24:663-76.

17. Nomi JS, Farrant K, Damaraju E, Rachakonda D, Calhoun VD, Uddin LQ. Dynamic functional network connectivity reveals unique and overlapping profiles of insula subdivisions. Hum Brain Mapp 2016;37:1770-87.

18. Li YO, Adali T, Calhoun VD. Estimating the number of independent components for functional magnetic resonance imaging data. Hum Brain Mapp 2007;28:1251-66.

19. Allen EA, Erhardt EB, Damaraju E, Gruner W, Segall JM, Silva RF, Havlicek M, Rachakonda S, Fries J, Kalyanam R, Michael AM, Caprihan A, Turner JA, Eichele T, Adelsheim S, Bryan AD, Bustillo J, Clark VP, Feldstein Ewing SW, Filbey F, Ford CC, Hutchison K, Jung RE, Kiehl KA, Kodituwakku P, Komesu YM, Mayer AR, Pearlson GD, Phillips JP, Sadek JR, Stevens M, Teuscher U, Thoma RJ, Calhoun VD. A baseline for the multivariate comparison of resting-state networks. Front Syst Neurosci 2011;5:2.

20. Fox MD, Snyder AZ, Vincent JL, Corbetta M, Van Essen DC, Raichle ME. The human brain is intrinsically organized into dynamic, anticorrelated functional networks. Proc Natl Acad Sci U S A 2005;102:9673-8.

21. Schmidt-Wilcke T. Neuroimaging of chronic pain. Best Pract Res Clin Rheumatol 2015;29:29-41.

22. Coppola G, Renzo AD, Petolicchio B, Tinelli E, Lorenzo CD, Serrao M, Calistri V, Tardioli S, Cartocci G, Parisi V, Caramia F, Piero VD, Pierelli F. Increased neural 
connectivity between the hypothalamus and cortical resting-state functional networks in chronic migraine. J Neurol 2020;267:185-91.

23. Hemington KS, Wu Q, Kucyi A, Inman RD, Davis KD. Abnormal cross-network functional connectivity in chronic pain and its association with clinical symptoms. Brain Struct Funct 2016;221:4203-19.

24. Filippi, M, Messina R. The Chronic Migraine Brain: What Have We Learned From Neuroimaging? Front Neurol 2020;10:1356.

25. Nugraha B, Gutenbrunner C, Barke A, Karst M, Schiller J, Schäfer P, Falter S, Korwisi B, Rief W, Treede RD. The IASP classification of chronic pain for ICD-11: functioning properties of chronic pain. Pain 2019;160:88-94.

26. Treede RD, Rief W, Barke A, Aziz Q, Bennett MI, Benoliel R, Cohen M, Evers S, Finnerup NB, First MB, Giamberardino MA, Kaasa S, Kosek E, Lavand'homme P, Nicholas M, Perrot S, Scholz J, Schug S, Smith BH, Svensson P, Vlaeyen JW, Wang SJ. A classification of chronic pain for ICD-11. Pain 2015;156:1003-7.

27. Ploner M, Sorg C, Gross J. Brain Rhythms of Pain. Trends Cogn Sci 2017;21:100-10.

28. Donaldson LF, Lumb BM. Top-down control of pain. J Physiol 2017;595:4139-40.

29. Kucyi A, Davis KD. The dynamic pain connectome. Trends Neurosci 2015;38:86-95.

30. Bushnell MC, Čeko M, Low LA. Cognitive and emotional control of pain and its disruption in chronic pain. Nat Rev Neurosci 2013;14:502-11.

31. Jiang Y, Oathes D, Hush J, Darnall B, Charvat M, Mackey S, Etkin A. Perturbed connectivity of the amygdala and its subregions with the central executive and default mode networks in chronic pain. Pain 2016;157:1970-8.

32. Shen W, Tu Y, Gollub RL, Ortiz A, Napadow V, Yu S, Wilson G, Park J, Lang C, Jung M, Gerber J, Mawla I, Chan ST, Wasan AD, Edwards RR, Kaptchuk T, Li S, Rosen B, Kong J. Visual network alterations in brain functional connectivity in chronic low back pain: A resting state functional connectivity and machine learning study. Neuroimage Clin 2019;22:101775.

33. Perkins M, Bruyne MD Giummarra MJ. A pain in the

Cite this article as: Zou Y, Tang W, Qiao X, Li J. Aberrant modulations of static functional connectivity and dynamic functional network connectivity in chronic migraine. Quant Imaging Med Surg 2021;11(6):2253-2264. doi: 10.21037/qims20-588 bud? Implications of cross-modal sensitivity for pain experience. Atten Percept Psychophys 2016;78:2348-56.

34. Hauck M, Metzner S, Rohlffs F, Lorenz J, Engel AK. The influence of music and music therapy on pain-induced neuronal oscillations measured by magnetencephalography. Pain 2013;154:539-47.

35. Moseley GL, Vlaeyen JW. Beyond nociception: the imprecision hypothesis of chronic pain. Pain 2015;156:35-8.

36. Cordier L, Diers M. Learning and Unlearning of Pain. Biomedicines 2018;6:67.

37. Davey CG, Pujol J, Harrison BJ. Mapping the self in the brain's default mode network. Neuroimage 2016;132:390-7.

38. Qiao L, Luo X, Zhang LJ, Chen AT, Li H, Qiu J. Spontaneous brain state oscillation is associated with self-reported anxiety in a non-clinical sample. Sci Rep 2020;10:19754.

39. Seeley WW, Menon V, Schatzberg AF, Keller J, Glover GH, Kenna H, Reiss AL, Greicius MD. Dissociable intrinsic connectivity networks for salience processing and executive control. J Neurosci 2007;27:2349-56.

40. Finn ES, Shen XL, Scheinost D, Rosenberg MD, Huang J, Chun MM, Papademetris X, Constable RT. Functional connectome fingerprinting: identifying individuals using patterns of brain connectivity. Nat Neurosci 2015;18:1664-71.

41. Zuo XN, Martino AD, Kelly C, Shehzad ZE, Gee DG, Klein DF, Castellanos FX, Biswal BB, Milham MP. The oscillating brain: Complex and reliable. Neuroimage 2010;49:1432-45.

42. Hermans EJ, Henckens MJ, Joels M, Fernandez G. Dynamic adaptation of large-scale brain networks in response to acute stressors. Trends Neurosci 2014;37:304-14

43. Smith EE, Jonides J. Neuroimaging Analyses of Human Working Memory. Proc Natl Acad Sci U S A 1998;95:12061-8.

44. Napadow V, LaCount L, Park K, As-Sanie S, Clauw DJ, Harris RE. Intrinsic brain connectivity in fibromyalgia is associated with chronic pain intensity. Arthritis Rheum 2010;62:2545-55. 